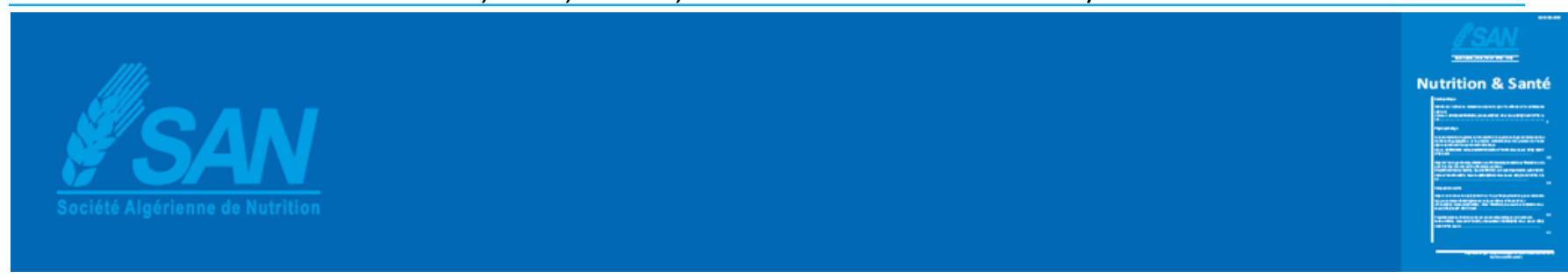

\title{
Additifs alimentaires
}

Effets aigus de l'exposition à la tartrazine (E102) sur le comportement et l'histologie d'un modèle biologique l'escargot terrestre Helix aspersa

Acute effects of tartrazine (E102) exposure on behavior, and histology of land snail Helix aspersa biological model

Smina AÏT HAMLET ${ }^{1}$, Samira BENSOLTANE ${ }^{1,2}$, Houria BERREBBAH ${ }^{1}$

${ }^{1}$ Laboratoire de Toxicologie Cellulaire, Faculté des Sciences, Université Badji-Mokhtar, Annaba, BP.12, 23000, Algérie. ${ }^{2}$ Faculté de Médecine, Université Badji-Mokhtar, Annaba, 23000, Algérie.

Auteur correspondant: smina1981@hotmail.fr

Reçu le 21 avril 2020, Révisé le 02 juin 2020, Accepté le 26 juin 2020

Résumé Introduction. La tartrazine est un additif alimentaire azoïque largement utilisé dans les industries pharmaceutique, cosmétique, alimentaire et autres. Objectif. Evaluer les effets de la tartrazine sur l'attitude à se déplacer et à se nourrir et les altérations histologiques au niveau de l'appareil digestif et rénal, après un traitement aigu, sur un modèle biologique l'escargot Helix aspersa. Matériel et Méthodes. Les escargots ont été exposés par voie orale et par contact à la farine contaminée par ce colorant pendant 24 heures. Ils sont répartis en deux groupes de 20 animaux chacun. Le groupe témoin a été nourri avec de la farine seule et le groupe expérimental avec de la farine traitée à la tartrazine $(200 \mathrm{mg} / \mathrm{g}$ de farine). Résultats. L'observation du comportement a révélé des effets inhibiteurs de la tartrazine sur la locomotion et la nutrition des escargots. Dans un $2^{\text {ème }}$ temps, l'examen histologique des intestins, de l'hépatopancréas et des reins des animaux traités a montré des structures tissulaires altérées avec une dégénérescence avancée des tubules digestifs, du tissu conjonctif et des fibres musculaires lisses des parois entourant les trois organes par rapport aux témoins. Conclusion. Cette étude suggère qu'une exposition aiguë à la tartrazine peut entraîner des troubles du comportement (locomoteur et nutritif), en plus des lésions tissulaires. Par conséquent, l'étude du comportement, appuyée par l'examen des altérations histologiques chez $\mathrm{H}$. aspersa peuvent être utilisés comme des biomarqueurs potentiels d'exposition à d'autres colorants azoïques.

Mots clés: Colorant alimentaire, Tartrazine, Helix aspersa, Comportement, Histologie, Intestins, Hépatopancréas, Rein

Abstract Introduction. Tartrazine is an azofood additive widely used in the pharmaceucal, cosmetic, food, and other industries. Objective. To assess the effects of tartrazine 
on the attitude to move and to eat, and histological changes in digestive, and renal system, after an acute treatment on a biological model the snail Helix aspersa. Material and Methods. Snails were exposed orally, and by contact to flour contaminated with this dye for 24 hours. Adult snails were divided into two groups of 20 animals each. The control group fed flour alone. The experimental group was administered orally with flour treated with tartrazine $(200 \mathrm{mg} / \mathrm{g}$ flour). Results. The observation of behaviour revealed inhibitory effects of tartrazine on locomotion, and nutrition of snails. Histological examination of intestines, hepatopancreas, and kidney of treated animals showed altered tissue structures, with advanced degeneration of digestive tubules, connective tissue, and smooth muscle fibers of the walls surrounding the three organs, in response to tartrazine, compared to controls. Conclusion. This study suggests that acute exposure to tartrazine may lead to disorders in locomotor, and nutritional behavior, in addition to tissue alterations. Therefore, this study on behavior, supported by histological changes in $H$. Aspersa can be used as potential biomarkers of exposure to other azodyes.

Keywords: Food dye, Tartrazine, Helix aspersa, Behavior, Hstology, Intestines, Hepatopancreas, Kidney

\section{Introduction}

L'emploi des colorants de synthèse et, notamment, des produits azoïques dans l'industrie pharmaceutique, cosmétique, alimentaire et textile ne cesse d'augmenter ces dernières décennies, du fait de certains avantages techniques et économiques. Cependant, la consommation d'un grand nombre de ces substances a favorisé l'émergence de nombreuses manifestations cliniques [1-3], ce qui a suscité de la méfiance, voire même, de l'inquiétude pour certaines d'entre elles [4], jugées comme étant douteuses [5-7] ou dangereuses [8-10]. Ces azo-molécules peuvent subir, dans l'organisme, des biodégradations, avec l'apparition d'amines aromatiques et/ou leurs dérivés, dont les effets cancérigènes ont toujours été redoutés [11].

Certains azoïques sont plus incriminés que d'autres et, particulièrement, la tartrazine. Cette substance présente un risque avéré d'hyperactivité chez l'enfant [2], ainsi qu'une allergie intense sur une fraction de consommateurs [12]. Elle présente, également, des effets cancérigènes possibles, y compris ses résidus de production [13, 14], mutagènes [15], neurotoxiques potentiels, car commercialisable sous forme de laque d'aluminium $[1,16]$, immunotoxiques [17], inhibiteurs de la respiration mitochondriale [18], suppresseurs de la reproduction [19] et d'éventuelles malformations morphologiques et squelettiques [2022].

La tartrazine est autorisée par l'Autorité Européenne de Sécurité des Aliments (AESA) et par le Comité
Scientifique de I'Alimentation Humaine (CSAH).Mais, certains pays l'ont interdite, comme les Etats Unis et la Suisse. La dose journalière acceptable (DJA) recommandée [23] est de 0 à $10 \mathrm{mg} / \mathrm{kg}$ de poids corporel/jour (Equivalent de 0 à $700 \mathrm{mg} / \mathrm{j}$, chez un homme de $70 \mathrm{~kg}$ ). La tartrazine est un colorant jaune artificiel, largement utilisé, pour teindre les bonbons, les jus, les boissons aromatisées sans alcool, les confitures, le chewing-gum, les gelées, la moutarde, les sodas, les glaces et les crèmes glacées, les médicaments et les cosmétiques [24]. Cependant, ses effets nocifs sur la santé restent à compléter, ce qui laisse ouvert le champ d'investigation.

Dans ce contexte, nous avons estimé, par une étude expérimentale, l'effet aigu de la tartrazinesur un modèle biologique, l'escargot terrestre Helix aspersa. Cette espèce de gastéropode est abondante dans la région Nord-Est de l'Algérie et très utilisée dans l'évaluation de l'impact des xénobiotiques sur les différentes fonctions physiologiques et comportementales [25-27]. Les escargots, comme $H$. aspersa, sont réputés par leur capacité à accumuler différentes catégories de contaminants chimiques dans leurs tissus, particulièrement, dans la glande digestive [27]. L'utilisation des altérations hépato-pancréatiques, comme biomarqueurs d'exposition aux xénobiotiques a été déjà investie [28-31].

La tartrazine induit des perturbations du comportement et des altérations tissulaires après une exposition prolongée ou répétée [32-35].

L'objectif de cette étude consiste en l'exploration des troubles du comportement et des effets histologi- 
ques possibles sur le système digestif (hépatopancréas, intestins) et rénal, après un traitement aigu à la tartrazine, chez $\mathrm{H}$. aspersa.

\section{Matériel et méthodes}

\section{Produits chimiques}

La tartrazine (trisodium 5-hydroxy-1-(4sulfonatophenyl)-4-(4-sulfonatophenylazo)-h-pyrazol3carboxylate, numéro de CAS 1934-21-0) utilisée dans cette étude est une poudre orange, provenant du marché (Algérie).

\section{Conduite de l'essai}

Des escargots $H$. aspersa adultes sont collectés d'un site non traité par les pesticides, situé dans la commune de Draa El Rich de la Wilaya d'Annaba dans la région Nord-Est d'Algérie. Ils sont ensuite transférés au laboratoire, où ils sont adaptés aux conditions contrôlées décrites par Gomot, [36] (température $20 \pm 2^{\circ} \mathrm{C}$, photopériode $18 \mathrm{~h}$ de lumière/6h d'obscurité, hygrométrie 80 à $90 \%$ ) pendant deux semaines. Ils sont nourris exclusivement de farine de blé. Les 40 escargots choisis ont une masse moyenne de $13,21 \pm 0,3 \mathrm{~g}$. Ils sont répartis en 2 groupes de 20 animaux chacun et maintenus dans des boites de polystyrène perforées de dimensions $25 \times 13,5 \times 16,5 \mathrm{~cm}$. Le $1^{\text {er }}$ groupe d'escargots, considéré comme témoin, est nourri de farine seule. Le $2^{\text {ème }}$ groupe reçoit de la farine traitée avec de la poudre de tartrazine en une dose unique de $200 \mathrm{mg} / \mathrm{g}$ pendant $24 \mathrm{~h}$, sous des conditions contrôlées de laboratoire citées auparavant.

\section{Estimation de la nourriture consommée}

Le poids moyen de la farine fournie aux escargots au début de l'essai $\left(P M_{0}\right)$ et celui de la farine, mesuré après $24 \mathrm{~h}\left(P \mathrm{M}_{1}\right)$ ont été évalués afin de déterminer le poids moyen de la farine consommée (PMC) et, par conséquent, le poids moyen de la tartrazine consommée ( $\mathrm{PM}$ T).

\section{Effets sur les comportements locomoteur et nutritif}

Au cours de l'exposition à la tartrazine, les effets de l'additif testé ont été évalués sur les comportements plus ou moins perturbés de $H$. aspersa. Certains comportements peuvent être observés, comme se nourrir normalement ou fuir la nourriture, l'activité des animaux (actif ou inactif) sur la nourriture. Ceci a été déjà rapporté par d'autres auteurs [37].

\section{Etude histologique}

Après ce traitement aigu, 4 spécimens choisis au hasard dans chaque groupe, sont sacrifiés par décapita- tion et leurs organes (intestin, hépatopancréas, rein) rapidement excisés pour l'étude histologique. Ces organes sont fixés dans du liquide de Bouin [38] pendant 3 jours. Les échantillons sont préparés aux analyses, après plusieurs étapes de rinçage à l'eau déminéralisée, déshydratation dans des bains d'alcool, à degré croissant et imprégnation dans un bain de paraffine [39]. Ensuite, les échantillons d'organes sont inclus dans la paraffine à l'aide de moules (barres de Leuckart), puis sectionnés à une épaisseur de $6 \mu \mathrm{m}$ par un microtome Leitz et collés sur des lames de verre,avec de l'eau albumineuse. Après déparaffinage et hydratation, les lames sont colorées à l'éosine-hématoxyline [40]. Les lames préparées sont observées au microscope (Leica DM LB2) photonique.

\section{Résultats}

\section{Estimation de la nourriture consommée}

Le Tableau I présente le PMc par les escargots traités qui est environ 7,1-fois plus faible que celui des témoins. Quant à la quantité de tartrazine moyenne consommée, elle est de $11,8 \mathrm{mg} /$ Escargot/24h.

Tableau I. Poids moyen (mg) de la farine et de la tartrazine consommé par les escargots

\begin{tabular}{lcc}
\hline & Témoins & Traités \\
\hline PMo $_{0}$ & 1000 & 1000 \\
PM1 $_{1}$ & 581 & 941 \\
PMc & 419 & 59 \\
PMT & 0 & 11,8 \\
\hline
\end{tabular}

$P M_{0}$ : poids moyen de farine au début; $P M_{1}$ : poids moyen à $24 \mathrm{~h}$; $P M_{C}$ : poids moyen consommé; $P M_{1}$ :poids moyen de tartrazine consommé.

\section{Effets sur les comportements locomoteur et nutritif}

Les escargots, exposés pendant $24 \mathrm{~h}$ à la tartrazine, ont montré la perte de la chimioréception, ils ne sont plus attirés par la nourriture, comparés aux témoins. Ils n'arrivent plus à se déplacer et se réfugient dans leurs coquilles, le diaphragme bombé, la saule pédieuse recroquevillée et arrivent à peine à bouger, même après lavage du corps avec l'eau de robinet (censée les faire sortir de leur coquille). Ils ne montrent aucune activité locomotrice et/ou nutritionnelle.

\section{Histopathologie}

La dissection des escargots, exposés au colorant azoïque, a montré une masse viscérale avec des organes remarquablement hypertrophiés (Fig. 1b) par rapport aux témoins (Fig. 1a).

Les œdèmes observés sont visibles à l'œil nu, particulièrement, au niveau des intestins (Fig. $\mathbf{2 b}$ et $\mathbf{c}$ ) par 
comparaison aux témoins (Fig. 2a).

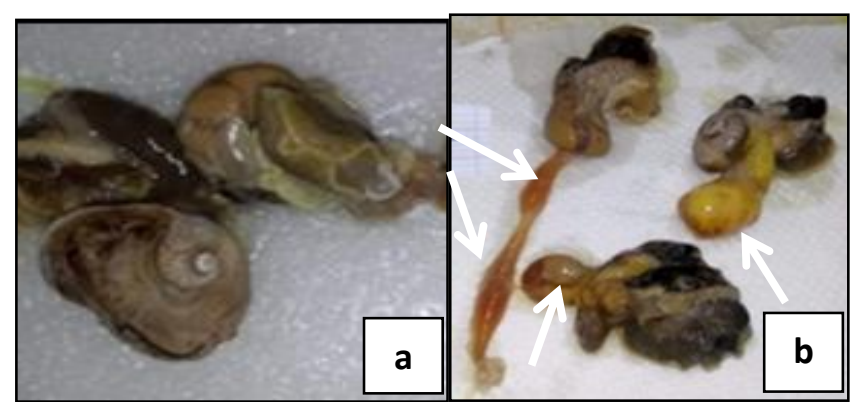

Fig. 1. Photographie de la masse viscérale

$\boldsymbol{a}$. Escargots témoins. $\boldsymbol{b}$. Escargots traités à la tartrazine montrant des œedèmes visibles au niveau des intestins. nectif. Les cellules digestives constituent la principale composante cellulaire de l'épithélium des tubules de la glande digestive et sont, relativement, polymorphes, selon le stade de la digestion [41]. Après exposition au colorant, l'examen histologique de l'hépatopancréas a montré une architecture tissulaire altérée avec une dégénérescence avancée des tubules digestifs (Fig. $\mathbf{4 b}$ et $\mathbf{c}$ ), un élargissement de l'espace intertubulaire connectif, des fibres musculaires très endommagées, une augmentation du foyer inflammatoire, notamment, au niveau de l'enveloppe conjonctive et musculaire entourant l'organe et la présence de débris musculaires.
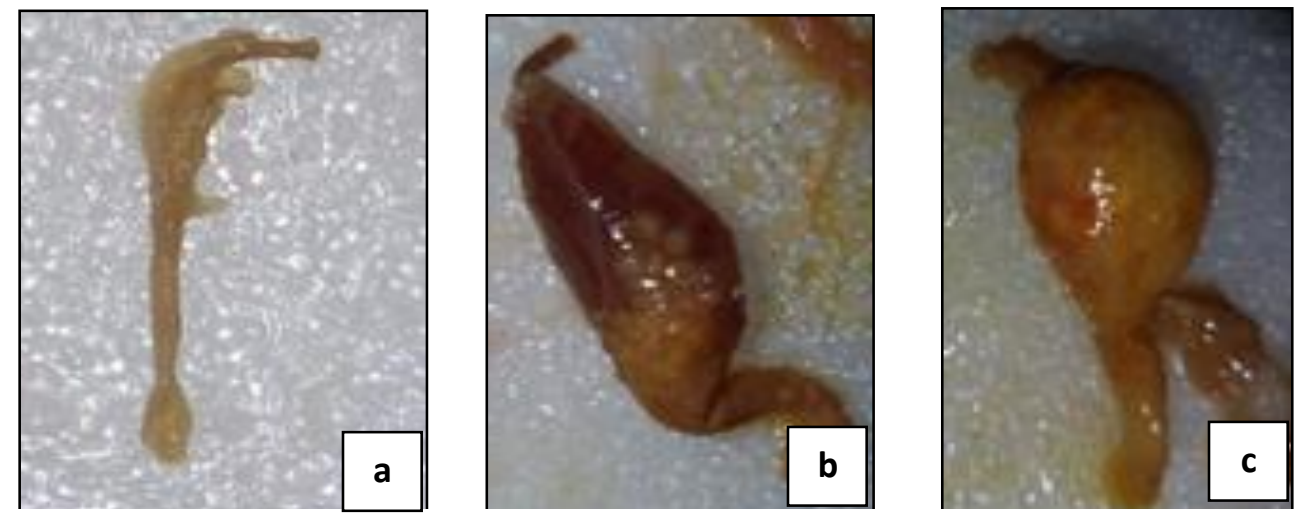

Fig. 2. Photographie des intestins

a. Escargots témoins. $\boldsymbol{b}$. et c. Escargots traités à la tartrazine

Une section histologique de l'intestin des escargots témoins a révélé un tube de diamètre constant (Fig. 3a), dont la paroi est localement soulevée en replis, de la lumière à la périphérie, elle est formée d'un épithélium simple et prismatique au sein duquel alternent des cellules de revêtement ciliées et des cellules glandulaires, d'une tunique de tissu conjonctif peu épaisse et de fibres musculaires lisses [41].

Après $24 \mathrm{~h}$ de traitement, l'examen histologique de l'intestin (Fig. $\mathbf{3 b}$ et $\mathbf{c}$ ) a révélé un aspect tissulaire global très altéré, avec un décollement net de la couche des fibres musculaires du tube digestif, des replis moins prononcés, des dégénérescences visibles du tissu conjonctif et des fibres musculaires lisses de la paroi. Une section histologique de l'hépatopancréas du groupe témoin est illustrée dans la Fig. 4a. Cette coupe montre que le tissu de la glande digestive est constitué, essentiellement, par la juxtaposition de nombreux tubules digestifs de différentes formes et tailles, séparés par l'espace inter-tubulaire. La lumière des tubules est bordée par un épithélium simple, associant plusieurs types cellulaires. Les tubules sont maintenus cohérents par le tissu inter-tubulaire con-
Une section histologique du rein (ou organe de Bojanus) des escargots témoins est illustrée dans la Fig. 5a. Cette coupe montre que la paroi rénale forme de nombreux replis, soutenus par des axes conjonctifs au sein desquels des lacunes hémolymphatiques sont présentes. Les replis sont tapissés d'un épithélium simple, prismatique, formé de cellules semblables les unes aux autres, les néphrocytes. Ces dernières possèdent un noyau basal associé à un cytoplasme chromophyle et leur région apicale est occupée par une volumineuse vacuole contenant une concrétion d'acide urique. Au contact de la lumière rénale, la membrane porte une bordure en brosse peu épaisse. L'uretère est sur une partie de son trajet accolé au rein. La paroi de l'uretère est formé de replis, plus au moins, développés dans sa région proximale, elle comporte au contact de la lumière un thulium simple et cubique formé de cellules munies d'une bordure en brosse apicale, l'épithélium repose sur du tissue conjonctif associé à de rares fibres musculaires lisses. L'uretère prend en charge l'urine produite [41]. Après traitement à la tartrazine, l'examen histologique de de l'organe de Bojanus (Fig. 5 b et c) 

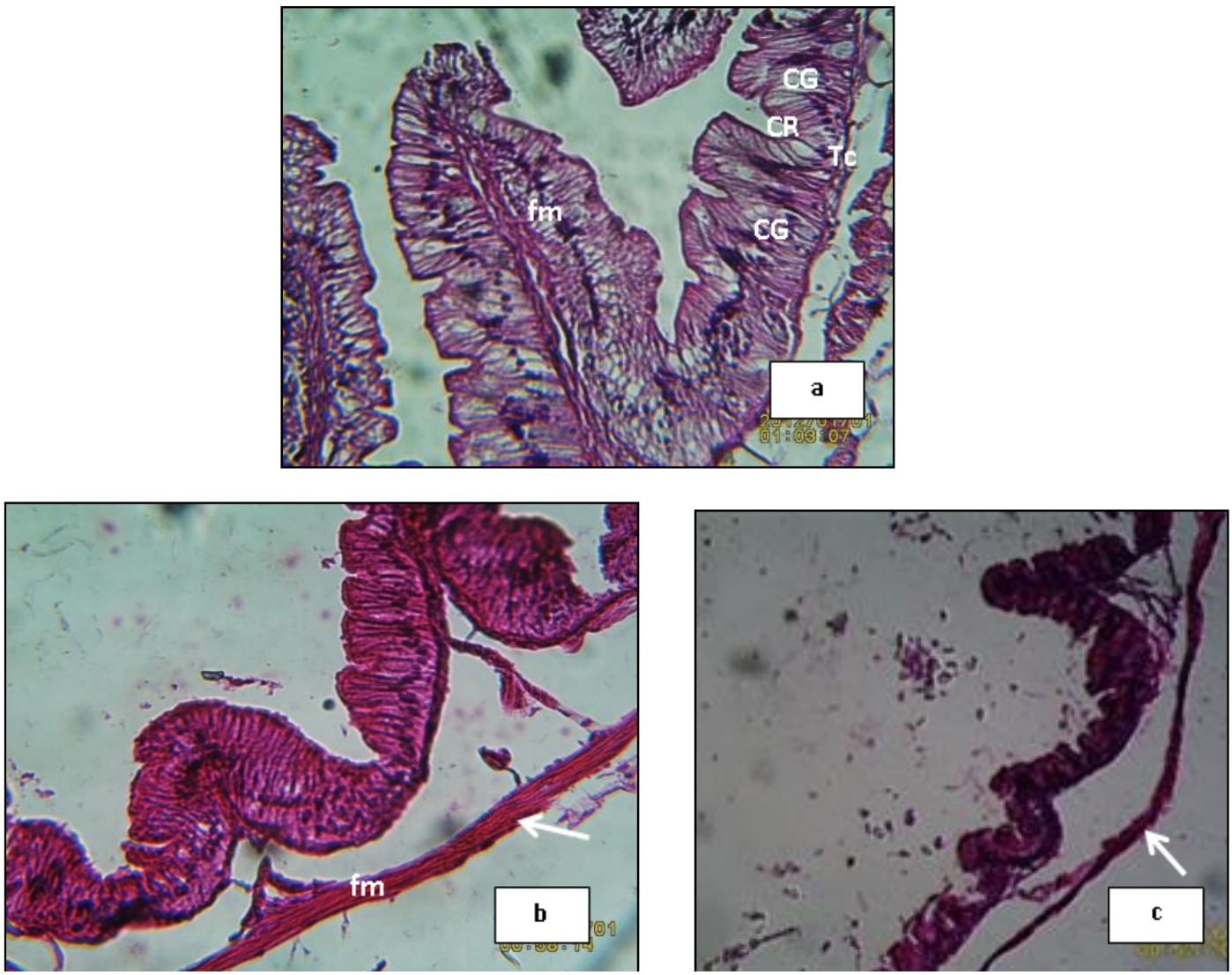

Fig. 3. Coupes histologiques des intestins de $H$. aspersa des groupes témoin et traité (après 24 heures d'exposition à la tartrazine) colorées à l'éosine-hématoxyline

$C G$, cellule glandulaire; $C R$, cellule de revêtement; $f m$, fibre musculaire lisse; $T c$, tissu conjonctif. a. Escargot témoin non traité montrant un tube de diamètre constant, formé d'un épithélium au sein duquel alternent les cellules glandulaires et les cellules de revêtement, puis une tunique de tissu conjonctif et une couche de fibres musculaires lisses. b. et c. Escargot traité avec $200 \mathrm{mg} / \mathrm{g}$ de tartrazine, montrant l'élargissement et le décollement net de la couche des fibres musculaires du tube digestif (flèches) et perte partielle des replis de la paroi. Grossissement 400x (a et b), grossissement 100x (c).
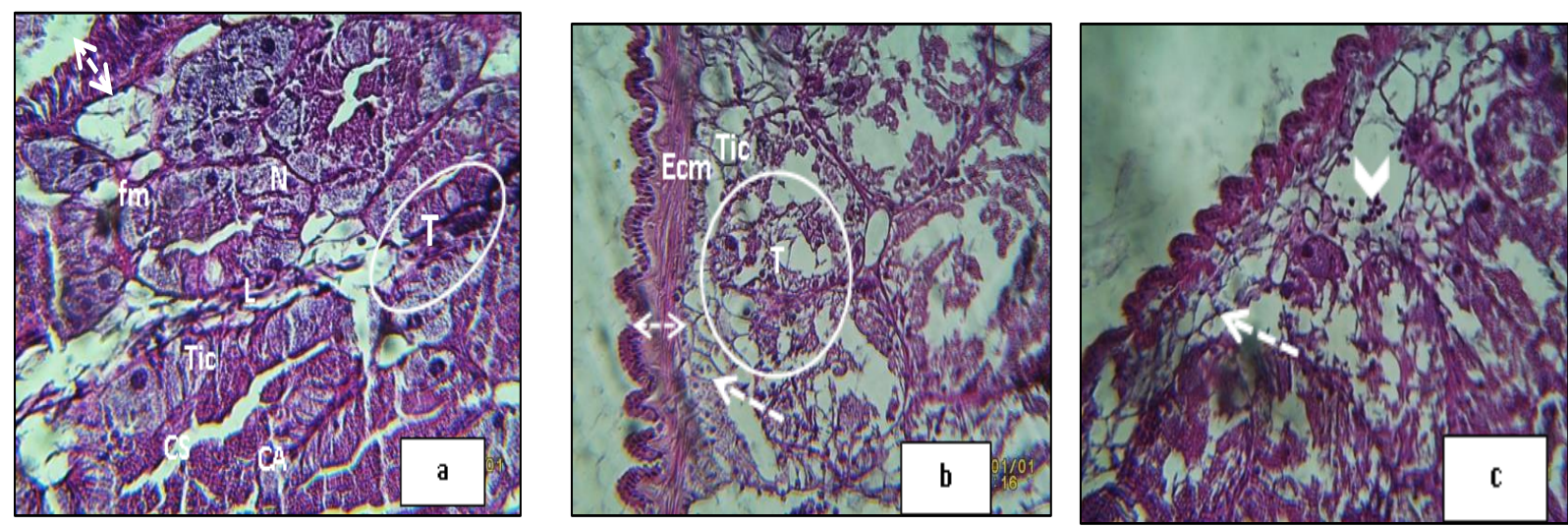

Fig. 4. Coupes histologiques de l'hépatopancréas de $H$. aspersa chez les groupes témoin et traité (après $24 \mathrm{~h}$ d'exposition à la tartrazine) colorées à l'éosine-hématoxyline.

Ecm, enveloppe conjonctive et musculaire; CS, cellule sécrétrice ; CA, cellule absorbante ; T, tubule digestif (cercle) ; Tic, tissu intertubulaire connectif ; L, lumière du tubule; $\mathrm{N}$, noyau; fm, fibre musculaire. a. Escargot témoin, non traité, montrant la juxtaposition de nombreux tubules digestifs de formes et de tailles diverses. b. et c. Escargot traité avec 200 $\mathrm{mg} / \mathrm{g}$ de tartrazine, montrant une dégénérescence des tubules digestifs (cercle), et du tissu intertubulaire connectif (flèche) avec altération et inflammation avancée de l'enveloppe hépatopancréatique (flèche à deux pointes) et présence de débrits cellulaires (pointe de flèche). Grossissement x400 ( $a, b$ et $\mathbf{c}$ ). 

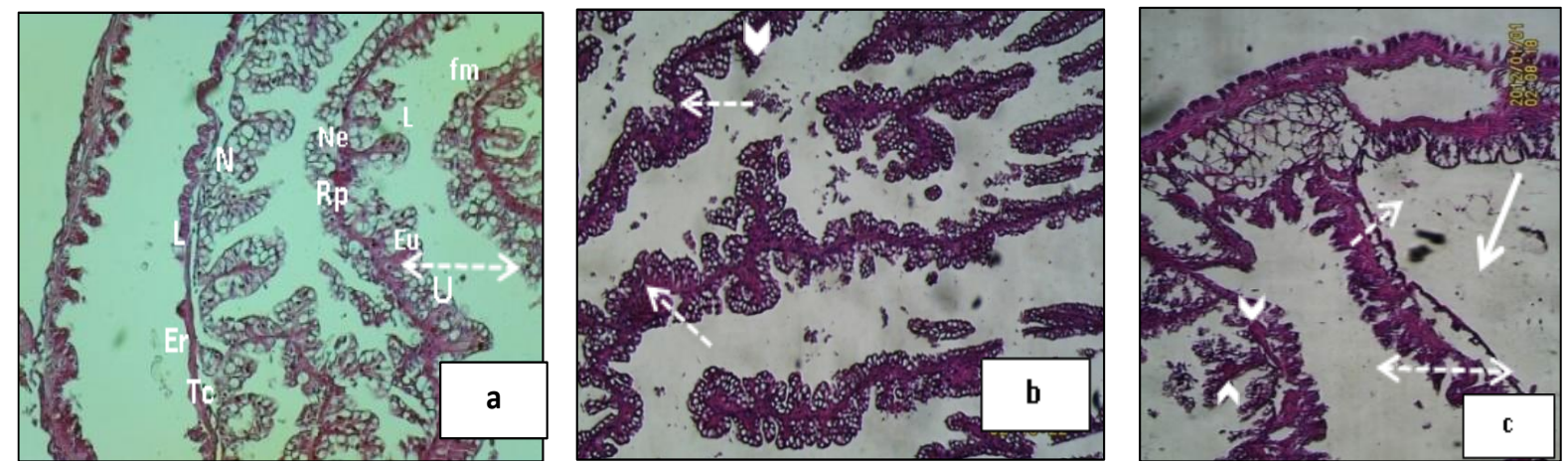

Fig. 5. Coupes histologiques du rein de $\boldsymbol{H}$. aspersa dans les groupes témoin et traité (après 24 h d'exposition à la tartrazine) colorées à l'éosine-hématoxyline

$U$, uretère (flèche à deux pointes);Ne, nephrocyte; Er, épithélium rénale; Eu, épithélium de l'uretère (cercle); L, lumière; Rp repli de la paroi; N, noyau; Tc, tissu conjonctif ; fm, fibre musculaire. a. Escargot témoin, non traité, montrant des replis tapissés d'un épithélium simple, formé de néphrocytes et l'urètre accolé au rein. b. et c. Escargot traité avec $200 \mathrm{mg} / \mathrm{g}$ de tartrazine, montrant une inflammation avancée du tissu conjonctif rénal et de la paroi de l'uretère (flèche pointillée) avec décollement visible de l'épithélium de l'uretère (flèche pleine) et présence de débrits cellulaires (pointe de flèche). Grossissement 100x (a, b et c)

a révélé une inflammation très avancée du tissu conjonctif de l'ensemble des replis rénaux et celui de l'uretère, en particulier, accompagnée d'un décollement visible de l'épithélium de l'uretère et l'envahissement de la lumière du tissu néphrotique par les débris cellulaires.

\section{Discussion}

Dans cette étude, les effets de la tartrazine, additif très utilisé en Algérie, ont été évalués sur le comportement et les atteintes histologiques de $H$. aspersa. Une dose de tartrazine, inférieure à la DJA chez un homme de $70 \mathrm{~kg}$, a été utilisée. Cependant, les escargots n'ont consommé qu'environ $1 / 17$ de cette DJA. Les troubles constatés sur le comportement locomoteur et nutritif des escargots suggèrent des effets neurotoxiques du colorant sur cette espèce de gastéropode. En effet, Osman [1] a rapporté que les colorants azoïques inhibent les cholinestérases (enzymes indispensables à la neurotransmission), en coupant l'acétylcholine, qui est nécessaire à la transmission de l'influx nerveux. De plus, des effets inhibiteurs similaires sur le comportement locomoteur des rats [42] ont déjà été rapportés.

De la même manière, la natation, l'orientation olfactive et le corriportement explorateur sont affectés par la prise de cet additif, chez les souris [43]. Cependant, Doguc et al., [44] ont rapporté que des ratons exposés à plusieurs colorants, y compris la tartrazine, présentaient une activité et une anxiétéconsidérablement accrues. De même, Kamel et El-lethey, [34] ont montré qu'il existe un réel lien de causalité entre la tartrazine et la modulation d'hyperactivité et d'anxiété chez le rat.

Sur le plan histologique, le colorant alimentaire azoï- que provoque des atteintes tissulaires visibles. Nos observations sont en accord avec celles de Ali et al., [45] qui ont rapporté des atteintes histologiques évidentes au niveau du foie et des reins, lors d'une exposition subchronique des rats à la tartrazine. Raposa et al., [15] ont montré que la tartrazine et, bien d'autres additifs alimentaires, contribuent à l'activation dose-dépendante des processus inflammatoires chez les souris. Dans la même optique, El-Borm et al., [46] ont rapporté la présence d'œdèmes et le déclenchement de phénomènes inflammatoires au niveau du cortex rénal et des hépatocytes, chez les ovo-embryons de Gallus domesticus. Aussi, Leo et al., [47] ont observé que les colorants azoïques favorisaient l'inflammation chez l'homme.

Les colorants alimentaires, comme tout xénobiotique, pénètrent dans le corps de l'escargot par voie orale et franchissent plusieurs barrières avant d'atteindre les intestins, puis I'hépatopancréas, organe de détoxification des xénobiotiques $[48,49]$. Ils peuvent être réversiblement absorbés par l'hépatopancréas, puis sont détoxifiés et, finalement, excrétés par les reins [50]. L'exposition aigue de $H$. aspersa à la tartrazine a entrainé des altérations cytologiques importantes au niveau de l'appareil digestif (intestins, hépatopancréas) et excrétoire (reins). En effet, la détérioration des cellules digestives entrainent, par conséquent, l'altération du tube digestif global, provoquée par l'ingestion de l'azoïque. Ces résultats sont en accord avec ceux de Amin et al., [32] et Khayyat et al., [9] qui ont constaté que la tartrazine, administrée aux rats expérimentaux, est à l'origine de sévères altérations de leurs tissus hépatiques et rénaux. Nos résultats corroborent, également, avec ceux d'ElDesoky et al., [51], qui ont rapporté, lors d'une étude toxicologique chronique chez des rats, que la 
tartrazine contenue dans l'alimentation a entrainé des atteintes histologiques du foie. Les mêmes dégénérescences ont été observées dans les hépatocytes de cobayes exposés à cet additif [52]. II en est de même pour Sasaki et al., [53], qui ont montré que les colorants alimentaires azoïques, dont la tartrazine, induisent des dommages de la molécule d'ADN, au niveau hépatique et rénal. Par ailleurs, Himri et al., [54] ont noté des dégénérescences remarquables, au niveau du foie et du rein, après un traitement subchronique à la tartrazine. Quant à El Golli et al., [55], ils ont prouvé que l'additif est capable d'induire, non seulement, des lésions hépatiques et rénales, mais aussi une hémato-toxicité et une immuno-toxicité, chez le rat adulte, en modifiant l'équilibre global entre les oxydants et les antioxydants. D'autre part, ElBorm et al., [46] ont constaté que l'exposition à la tartrazine des embryons de Gallus domesticus entrainait des effets dégénératifs et inflammatoires sur les deux organes. Les dommages structuraux visibles, en réponse à l'absorption de cet azo-colorant, ont été, aussi, mis en évidence par Elbanna et al., [35], non seulement, chez les rats traités par la tartrazine au niveau du foie, des reins, de la rate et de l'intestin grêle, mais aussi, chez ceux traités par les métabolites de cet azoïque. Les lésions observées sont le reflet du traitement par ce colorant ou de ces métabolites. Cependant, la sévérité ainsi que la fréquence de ces lésions intègrent les effets de nombreux facteurs, comme la dose administrée, le temps d'exposition, les facteurs génétiques et l'espèce considérée [56].

\section{Conclusion}

Au terme de ce bio-essai, nous pouvons conclure que l'administration de la tartrazine, en dose unique de $200 \mathrm{mg}$ pour seulement $24 \mathrm{~h}$, engendre des perturbations du comportement et des anomalies visibles au niveau du système digestif et rénal de l'escargot $H$. aspersa. Les altérations révélées à la dose testée sont à compléter par les effets de l'exposition aux doses répétées, ou aux coktails.

Il est évident que la tartrazine présente un risque pour la santé. Ces données pourraient justifier une tentative de limitation d'incorporation de ce colorant dans nos aliments.

\section{Conflit d'intérêts}

Les auteurs déclarent n'avoir aucun conflit d'intérêt en relation avec cet article.

\section{Références}

1. Osman MY. Synthetic Organic Food Colouring Agents and TheirDegradedProducts: Effects on Human and Rat Cholinesterases. Br J Biomed Sci 2004; 61(3):128-32.

2. McCann D., Barrett A., Cooper A., Crumpler D., Dalen L., Grimshaw K. et al. Food additives and hyperactive behaviorin 3year-old and 8/9-yearold children in the community: arandomized, double-blinded, placebo-controlled trial. Lancet 2007; 370(9598):1560-7.

3. Park S. Association Between Dietary Behaviors and Attention-Deficit/Hyperactivity Disorder and Learning Disabilities in School-Aged Children. Psychiatry Res 2012;198(3):468-76.

4. Gouget C. Additifs Alimentaires Danger. Le guide indispensable pour ne plus vous empoisonner. Escalquens : éd. Chariot d'or; 2012, p.1-45.

5. Park HW., Park CH., Park SH., Park JY. Dermatologic Adverse Reactions to 7 Common Food Additives in Patients with Allergic Diseases: A DoubleBlind, Placebo-Controlled Study. J Allergy Clin Immunol 2008 ; 121(4): 1059-62.

6. Doguc DK. Are There Any Remark able Effects of Prenatal Exposure to Food Colourings on Neurobehaviour and Learning Process in Rat Offspring? Nutr Neurosci 2015 ; 18(1): 12-21.

7. Kim KM. Associations between Attention-Deficit/Hyperactivity Disorder Symptoms and Dietary Habits in Elementary School Children. Appetite 2018;127:274-9.

8. Kashanian S. DNA Binding Studies of Tartrazine Food Additive. DNA Cell Biol 2011 ;30(7):499-505.

9. Khayyat L., Essawy A., Sorour J., Soffar A. Tartrazine induces structural and functional aberrations and genotoxic effects in vivo. Peer J 2017 ;5: e3041.

10. Bhatt D. Tartrazine Induced Neurobiochemical Alterations in Rat Brain Sub-Regions. Food Chem Toxicol 2018 ; 113: 322-7.

11. Vobecky JS. Les additifs alimentaires: La controverse. Can Fam Physician 1982;28:746-51.

12. Inomata N., Osuna H., Fujita H., Ogawa T., Ikezawa Z. Multiple chemical sensitivies following intolerance to azodye in sweets in a 5-years-old girl. Allergol Int 2006;55:203-5.

13. Watab T., Ozawa N., Kobayashi F., Kurata H. Reduction of sulphonated water-soluble azodyes by micro-organismes from human faeces. Food CosmetToxicol 1980;18:349-52.

14. OuldElhkim M., Héraud F., Bemarh N., Gauchard F., Lorino T., Lambré C. et al. New considerations 
regarding the risk assessement on Tartrazine; An update toxicological assessment, intolerance reactions and maximum theoretical daily intake in France. Regul Toxicol Pharmacol 2007;47:308-16.

15. Raposa B., Pónusz R., Gerencsér G., Budán F., Gyöngyi Z., Tibold A. et al. Food Additives: Sodium Benzoate, Potassium Sorbate, Azorubine, and Tartrazine Modify the Expression of NFkB, GADD45 $\alpha$, and MAPK8 Genes. Physiol Int 2016 ; 103(3): 334-43.

16. Lau K. Synergistic Interactions Between CommonIy Used Food Additives in a Developmental Neurotoxicity Test. Toxicol Sci 2006;90(1):178-87.

17. Stevenson J., Sonuga-Barke E., McCann D., GrimshawK., Parker KM., Rose-Zerilli MJ. et al. The role of histamine degradation gene polymorphisms in moderating the effects of food additives on children's ADHD symptoms. Am J Psychiatry 2010;167(9):1108-15.

18. Reyes FG., Valim MF., Vercesi AE. Effect of Organic Synthetic Food Colours on Mitochondrial Respiration. Food Addit Contam 1996;13(1):5-11.

19. Meghapriya A., Kishori B. Tartrazine, a male reproductive supressor in adult albino rats. Int $J$ Life Sci Res 2019;7(3):1-10.

20. Boussada M., Lamine JA., Bini I., Abidi N., Lasrem M., El-Fazaa S., El-Golli N. Assessment of a subchronic consumption of tartrazine (E102) on sperm and oxidative stress features in Wistar rat. Int Food Res J 2017;24:1473-81.

21. Hashem MM., Abd-Elhakim YM., Abo-EL-Sooud K., Eleiwa MME. Embryotoxic and Teratogenic Effects of Tartrazine in Rats. Toxicol Res 2019;35(1); 75-81.

22. El-Borm HT. Badawy GM., El-Nabi SH., El-Sherif WA., Atallah MN. The ameliorative effect of curcumine xtract on the morphological and skeletal abnormalitie sinduced by sunset yellow and tartrazine in the developing chick embryo Gallus domesticus. Heliyon 2020;6: e03305.

23. WHO (Word Health Organisation). Evaluations of the joint FAO/WHO Expert Committee on Food Additives :Tartrazine.2019; ;ttps://apps.who.int/ food-additives-contaminants-jecfadatabase/ chemical.aspx?chemID $=3885$.

24. Gallen C., Pla J. Allergie et intolérance aux additifs alimentaires. Rev Fr Allergol 2013;53:9-18.

25. Gomot-de Vaufleury A. Standardized growth toxicity testing $(\mathrm{Cu}, \mathrm{Zn}, \mathrm{Pb}$, and Pentachlorophe-nol) with Helix aspersa. Ecotoxicol Environ Saf 2000; 46:41-50.

26. Snyman RG., Reinecke AJ., Reinecke SA. Field application of a lysosomal assay as biomarker of copperoxychloride exposure, in the snail Helix aspersa. Bull Envir Contam Toxicol 2002;69:11722.

27. Regoli F., Gorbi S., Fattorini D., Tedesco S., Notti A., Machella N. et al. Use of the land snail Helix aspersa as sentinel organism for monitoring ecotoxicologic effects of urban pollution: an integrated approach. Environ Health Perspect 2006;114:63-9.

28. Marigomez IA., Kortabitarte M., Dussart GBJ. Tissue level biomarkers in sentinel slugs as cost effective tools to assess metal pollution in soils. Arch Environ ContamToxicol 1998;34:167-76.

29. Radwan MA., Essawy AE., Abdelmeguied NE., Hamed SS., Ahmed AE. Biochemical and histochemical on the digestive gland of Eobania vermiculata snails treated with carbamate pesticides. Pestic Biochem Physiol 2008;90:154-67.

30. Aït Hamlet S., Djekoun M., Smati M., Semassel A., Djekoun Bensoltane S., Berrebbah H. Histopathological effects of neonicotinoid insecticide in the hepatopancreas of terrestrial gastropod Helix aspersa. Fresenius Environ Bull 2014;23(11): 3041-7.

31. Ait Hamlet S., Djekoun M., Bensoltane S., Berrebbah $\mathrm{H}$. Effets du thiaméthoxame et de la téfluthrine sur les biomarqueurs physiologiques de l'escargot terrestre Helix Aspersa. Rev Agrobiol 2019;9(2):1491-8.

32. Amin KA., Abdel Hameid H., Abd Elsttar AH. Effect of food azodyes tartrazine and carmoisine on biochemical parameters related to renal, hepaticfunction and oxidative stress biomarkers in young male rats. Food ChemToxicol 2010 ; 48(10): 29949.

33. Gao Y., Li C., Shen J., Yin H., An X., Jin H. Effect of food azodye tartrazine on learning and memory functions in mice and rats, and the possible mechanisms involved. J Food Sci 2011;76(6):125-9.

34. Kamel MM., El-lethey HS. The potential health hazard of tartrazine and levels of hyperactivity, anxiety-like symptoms, depression and anti-social behaviour in rats. J Am Sci 2011;7(6):1211-8.

35. Elbanna K., Sarhan OM., Khider M., Elmogy M., Abulreesh HH., Shaaban MR. Microbiological, histological, and biochemical evidence for the adverse effects of food azodyes on rats. J Food Drug Anal 2017;25(3):667-80.

36. Gomot A. Contribution à l'étude de la croissance d'escargots du genre Helix: influence de facteurs de l'environnement, nutrition et composition biochimique, contrôle neuroendocrine. Doctorat Sciences de la Vie. Université de Besançon- 
France; 1994 ;258p. http://www.theses.fr/1994BESA2046.

37. Chevallier H. L'élevage des Escargots. Production et Préparation du Petit-Gris. $2^{\text {ème }}$ éd. Ed. du point vétérinaire, Maison-Alfort, Paris; 1992, 144p.

38. Preece A. A manual for histologic technicians. Little, Brown and Company, Boston. 1972, 428p.

39. Odendaal JP., Reinecke AJ. Quantifying histopathological alterations in the hepatopancreas of the woodlouse Porcelliolaevis (Isopoda) as a biomarker of cadmium exposure. Ecotox Environ Safe 2002;56(2):319-25.

40. Martoja R., Martoja PM. Initiation aux techniques de l'histologie animale. Masson et Cie, Paris VI; 1967, 347p.

41. Heusser S., Dupuy HG. Synthèse de la structure tissulaire à la réalisation des fonctions chez les Gastéropodes Pulmonés (I) Eléments d'histologie et de physiologie des espèces Helix aspersa et Helix pomatia. Folia Chonchiliologica 2011;10:325.

42. Tanaka T. Reproductive and Neurobehavioural Toxicity Study of Tartrazine Administered to Mice in the Diet. Food Chem Toxicol 2006;44(2):17987.

43. Tanaka T., Takahashi O., Oishi S., Ogata A. Effects of tartrazine on exploratory behaviour in a threegeneration toxicity study in mice. Reprod Toxicol 2008;26:156-63.

44. Doguc DK., Ceyhan BM., Ozturk M., Gultekin F. Effects of Maternally Exposed Colouring Food Additives on Cognitive Performance in Rats. Toxicol Ind Health 2013;29(7):616-23.

45. Ali A., Sherein Abdelgayed SA., EL-Tawil SO., Bakeer MA. Toxicological and histopathological studies on the effect of tartrazine in male albino rats. Int J Biol Biomol Agric Food Biotechnol Eng 2016 ; 10: 491-6.

46. El-Borm H., Badawy GM., El-Nabi SH., El-Sherif WA., Atallah MN. Efficacy of curcumin on sunset yellow and tartrazine induced hepatotoxicity and nephrotoxicity in the chick embryo Gallus domesticus. Eur J Pharm Med Res 2019;6(11):48-64.

47. Leo L., Loong C., Ho XL., Raman MFB., Suan MYT., Loke WM. Occurrence of Azo Food Dyes and their
Effects on Cellular Inflammatory Responses. $\mathrm{Nu}$ trition 2018;46: 36-40.

48. Triebskorn R., Künast C. Ultrastructural changes in the digestive system of Deroceras reticulatum (Mollusca: Gastropoda) induced by lethal and sublethal concentrations of the carbamate molluscicide Cloethocarb.Malacologia 1990;32:89-106.

49. Frias-Espericueta MG., Abad-Rosales S., NevárezVelázquez AC., Osuna-López I., Páez-Osuna F., Lozano-Olvera R. Histological effects of a combination of heavy metals on pacific white shrimp $\mathrm{Li}$ topenaeusvannamei juveniles. Aquat Toxicol 2008;89:152-7.

50. Ishaaya I. Biochemical sites of insecticide action and resistance. Springer-Verlag Berlin Heidelberg; 2001, 283-321.

51. El-Desoky G., Abdel-Ghaffar A., Al-Othman ZA., Habila MA., Al-Sheikh YA., Ghneim HK. et al. Cucumin protects against tartrazine mediated oxidative stress and hepatotoxicity in male rats. Eur Rev Pharmacol Sc i2017;21:635-45.

52. Rus VGC., Miclaus V., Mihalca A., Nadăş GC. Comparative toxicity of food dyes on liver and kidney in guinea pigs: histopathological study. Ann Rom Soc Cell Biol 2009;15(1):161-5.

53. Sasaki YF., Kawaguchi S., Kamaya A., Ohshita M., Kabasawa K., Iwama K. et al. The comet assay with 8 mouse organs: results with 39 currently used food additives. Mutat Res 2002;519:103-9.

54. Himri I., Bellahcen S., Souna F., Belmekki F., Aziz M., Bnouham M. et al. A 90-day Oral Toxicity study of tartrazine, a synthetic food dye, in Wistar rats. Int J Pharm Pharm Sci 2011;3(3):159-69.

55. El Golli NN., El-Bini ID., Jrad AL., Boudali I., Nasri B., Belhadjhmida N., El-Fazaa S. Toxicity induced after subchronic administration of the synthetic food dye tartrazine in adult rats, role of oxidative stress. Recent Adv Biol Med 2016;2:20-8.

56. Adams SM., ShugartLR., Southworth GR., Hinton $D E$. Application of bioindicators in assessing the health of fish populations experiencing contaminant stress. Biomarkers Environ Contamin (Eds. McCarthy JF., Shugart LR). Lewis Publishers, CRC Press, Boca Raton; 1990 ;333-353. 